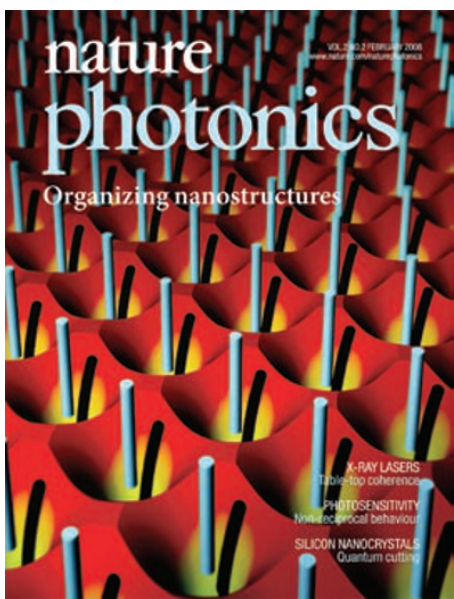

Cover story

Vol.2 No.2 February 2008

\begin{abstract}
The ability to separate and arrange nanowires of differing composition is much sought after in the world of nanotechnology. Now Ming Wu and co-workers from the University of California and Lawrence Berkeley National Laboratory have demonstrated optoelectronic tweezers that are able to separate individual metallic and semiconducting nanowires with diameters smaller than $20 \mathrm{~nm}$, and orient and manipulate arrays of silver nanowires. The approach relies on projecting a pattern of light onto a sheet of photoconductive material sandwiched between transparent electrodes. On application of a voltage, nanowires in a layer of liquid between the electrodes become polarized, orient themselves with the electric field, and are trapped by the field gradient of the light pattern, following it as it is scanned. [Letter p85; News \& Views p69]
\end{abstract}

\section{QUANTUM CUTTING}

Improving the performance of silicon solar cells is clearly an important goal with many potential benefits for energy generation in the future. The inefficiency is partly due to the fact that in conventional solar cells, short-wavelength, highly energetic single photons only generate a single electron-hole pair, and the excess energy is lost as unwanted heat. Now, scientists in Europe have reported that a scheme called quantum cutting may help by splitting such energetic photons into two or more photons of lower energy that are each able to generate their own electron-hole pair. In this issue, Dolf Timmerman and colleagues experimentally demonstrate this technique in silicon nanocrystals for the first time by using erbium ions and neighbouring nanocrystals to detect the effect. In theory the approach could be used to increase the efficiency of solar cells beyond the Shockley-Queisser limit, which governs the performance of conventional designs. [Article p105; News \& Views p72; Interview p122]

\section{THE IMPORTANCE OF DIRECTION}

Although photosensitivity is a commonly used technique for modifying the properties of a medium, it has always been assumed that the effect is independent of the direction of motion of the laser beam inducing the effect. Now, a team of scientists from the University of Southampton in the UK and the University of Joensuu in Finland have shown that this is in fact not the case. In experiments where femtosecond laser pulses are shone onto $z$-cut lithium niobate, Weijia Yang and colleagues observe that the interaction between the laser beam and the material changes when the direction of the writing laser beam is reversed. Their explanation for this 'non-reciprocal photosensitivity' is the crystal's anisotropy, which results in asymmetric heat flow. The team hold the opinion that this knowledge could have applications in materials processing and optical data storage.

[Article p99; News \& Views p73]

\section{EXTREME CONVENIENCE}

Convenient sources of coherent

13-nm light are required for imaging applications and extreme-UV lithography systems that are being developed to fabricate future generations of semiconductor integrated circuits. It now appears that table-top X-ray lasers may be a promising source of such radiation. In this issue, Jorge Rocca and co-workers from Colorado State University report the first experimental demonstration of table-top X-ray lasers that operate with wavelengths below $20 \mathrm{~nm}$ and have a high degree of spatial and temporal coherence. Their source operates by injecting high-harmonic seed pulses from a Ti:sapphire laser into a lasercreated plasma and amplifying the seed pulses by the 18.9-nm and 13.9-nm transitions of molybdenum and silver atoms, respectively.

[Letter p94; News \& Views p67]

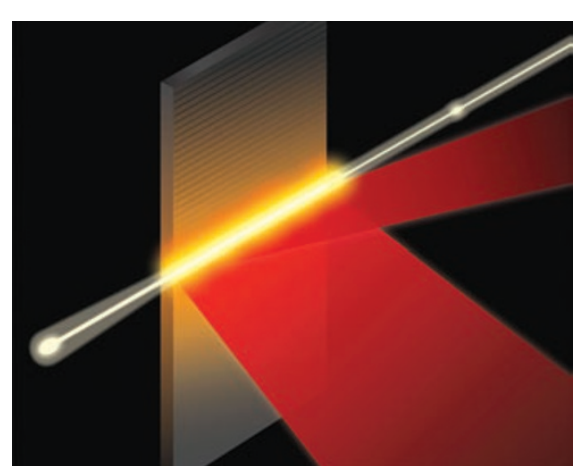

Jorge Rocca and co-workers demonstrate a compact extreme-UV laser that is spatially and temporally coherent.

\section{SCATTER SOLUTION}

Imaging the interior of biological samples using infrared or visible light is a challenge owing to strong optical scattering, which scrambles the path of the photons propagating through tissue. Now Changhuei Yang and co-workers show that optical phase conjugation can be used to force scattered light fields to retrace their trajectory through a sample and recover the original light field. In this issue, the researchers experimentally demonstrate the idea using green $(532 \mathrm{~nm})$ light and a 0.69 -mm-thick sample of chicken breast. The approach could prove useful for medical imaging, and it may also have applications for photodynamic therapy, where it can be used to efficiently guide photons to excite dyes located at specific points in tissue. Interestingly, such phase-conjugation techniques are already proving promising for medical therapies in the microwave and ultrasound regimes to enhance the delivery of energy to targets. [Article p110; News \& Views p71]

\section{LIGHT LOCALIZATION}

Developers of optical circuitry that uses compact waveguide structures to slow light and create all-optical memories and buffers may need to be careful as they scale their designs to larger and more complex configurations. Shayan Mookherjea and colleagues from the University of California, San Diego, have now observed that in a long chain of coupled resonators light can become localized. Their experiments with a onedimensional chain of silicon nanophotonic waveguides show that the localization leads to locally trapped light for wavelengths near the band edge. They conclude that disorder in the structures due to fabrication imperfections affects their optical transmission characteristics, and that the greater the disorder, the shorter the lengths required to induce the localization.

[Letter p90; News \& Views p75] 